\title{
Recent Development on Synthesizing and Characterizing Food-Grade Microemulsions
}

\author{
Sun Chang-Bao
College of Food and Biological Engineering. Qiqihar
University
Qiqihar, China \\ e-mail: sunchangbao@126.com
}

\author{
Wu Hong-Yan* \\ College of Food and Biological Engineering. Qiqihar \\ University \\ Qiqihar, China \\ Corresponding author: Wu Hong-Yan, Ph.D., \\ Professor, Tel: +86_452_2438743 \\ e-mail: hljwuhongyan@163.com
}

\author{
Li Ang \\ College of Food Science, Northeast Agricultural \\ University, \\ Harbin, China \\ e-mail: liang621liang@126.com
}

\begin{abstract}
Microemulsions have great applications in food products. However, due to the complex attributes of microemulsions, it requires various techniques to synthesize and characterize microemulsions. This includes studies of the phase behavior, size of the microemulsion droplets. This paper reviews different technologies used in preparing and characterizing food-grade microemulsions. The list of technologies summarized in this paper provides insights on future studies of food-grade microemulsions development.
\end{abstract}

Keywords-microemulsions; food-grade; characterization methods; synthesizing; technologies

\section{INTRODUCTION}

Microemulsions are mixed dispersion system composed of water, oil and surfactant in suitable proportions. Microemulsions are clear, transparent or translucent, and thermodynamically stable ${ }^{[1]}$. Microemulsion system has been more and more applications in the petrochemical, materials science, analytical chemistry, biomedical, and environmental protection. In 1943, Hoar and Schulman ${ }^{[2]}$ firstly discovered the structure of microemulsions. In 1959, Schulman et al. ${ }^{[3]}$ used the word "microemulsion" for the first time, which was to define a mixture of nanoscale particles (particle size 10-100 nm). In April 1990, the first international conference was organized on both microemulsions and food (in the Boston meeting of the American Chemical Society 199th Annual Meeting). Since then, microemulsions applied research on food products has made considerable progress. Microemulsions have many advantages: transparent or translucent, small viscosity, good fluidity, small and uniform particle size, wide dispersion, long-term storage, thermodynamically stable, rapid absorption, and improved bioavailability ${ }^{[4]}$. Microemulsions have great applications in food products, and can be used as protective solution for chemically unstable substances. Due to the complex attributes of microemulsions, it requires various techniques to synthesize and characterize microemulsions. This includes studies of the phase behavior, size of the microemulsion droplets. This paper reviews different technologies used in preparing and characterizing food-grade microemulsions.

\section{PREPARATION OF MicROEMUlsions}

Microemulsions are capable of spontaneously formed. However, in some cases, external energy is needed to overcome the energy barriers to accelerate the formation of microemulsions. The following three methods are major protocols to prepare microemulsions ${ }^{[5]}$.

\section{A. Hydrophilic - Lipophilic Balance (HLB)}

Surfactants are amphiphilic molecules with hydrophilic groups and lipophilic groups, degree of balance between hydrophilic and lipophili group define as HLB value. HLB value of the surfactant has a great influence on the formation of microemulsions. HLB value of surfactants for water-in-oil microemulsion is between 4 and 7. HLB value of surfactants are formed oil-in-water microemulsion is between 8 and 18. When the HLB values of the surfactant and oil phase are closer, it is easier to form the microemulsion $^{[6]}$. In order to allow additional steric flexibility for surfactant rearrangements, a cosurfactant (typically a short-chain alcohol) is combined with the surfactant. Co-surfactant can also play a regulatory role in forming microemulsions.

\section{B. Phase Inversion Temperature (PIT)}

For a particular microemulsion system, there is a narrow temperature range. Above this temperature, surfactant is dissolved in the oil phase, whereas below this temperature, surfactant is dissolved in the aqueous phase. With the temperature gradually increased, the system from the $\mathrm{O} / \mathrm{W}$ transform into a $\mathrm{W} / \mathrm{O}$ type, the temperature of the phase inversion occur is called the phase inversion temperature. PIT is the balance temperature of surfactant hydrophilic-lipophilic properties at the interface ${ }^{[7]}$. 
Temperature changes have great influence on the hydrophilic of surfactant. If the surfactant is in this state, the hydrophilic lipophilic achieve the right balance, with a strong solubilizing power and ultra-low interfacial tension. If temperature is lower than PIT, emulsion will form an oil-in-water microemulsion; if temperature is higher than PIT, emulsion will form a water-in-oil microemulsion; if temperature equals PIT, microemulsion will form bicontinuous microemulsions. PIT method is more specific and accurate compared to HLB value method.

\section{Salinity scanning}

Salinity scanning method is to influence salinity content of microemulsion. When the ratio of oil, water and surfactant are defined, if salinity increases from low to high, three states will be formed, namely Winsor I , Winsor II , WinsorIII. This method is mainly used for microemulsions with ionic surfactant .

\section{MiCROEMULSIONS CHARACTERIZATION METHODS}

Microemulsions are complex systems. Detailed understanding of microemulsions requires comprehensive characterization of different attributes of the systems.

\section{A. Dissipative Particle Dynamics (DPD)}

DPD method provides a powerful tool to simulate the behavior of complex fluids at mesoscopic scale to measure microstructure change ${ }^{[8,9]}$. DPD method evaluates both the microscopic interactions between substances, and the hydrodynamic behavior of cluster of matters. DPD method can be used to simulate the microstructure change in the process of formation of microemulsion system. Yinong Tian et al. ${ }^{[10]}$ investigated the dynamical process of microemulsion system formation using DPD method. The results showed that the microstructure and droplet shape changed during water phase solubilizing.

\section{B. Electron Microscopy}

Electron microscopy is an important technology to characterize microemulsion. Electron microscopy is highly sensitive and with fast processing time, and can be used to observe particle size, shape, and state of microemulsions. Transmission electron microscope (TEM) with negative staining is the most widely used electron microscopy protocol. The whole process from sample negative staining to obtaining results takes only ten minutes ${ }^{[11]}$. Chu B-S et al. ${ }^{[12]}$ measured particle size distribution and microstructure of microemulsion using Transmission electron microscope. The Transmission electron microscope results confirmed the results from Dynamic light scattering, showing that $\beta$-carotene particles were spherical, with an average diameter of $20 \mathrm{~nm}$.

\section{Dynamic light scattering (DLS)}

DLS is a conventional characterization method in nanotechnology to measure the change of the scattering light intensity fluctuation of sample to obtain sample particle size information ${ }^{[13]}$. DLS technology is fast, accurate and with good reproducibility. This method is not destructive, but there is a limit on the sample concentration. Several studies applied DLS to study the stability of microemulsion system by evaluating particle size and polydispersity ${ }^{[14,15]}$. Shukla A et al. ${ }^{[16]}$ studied the diffusion behavior of the oil-in-water drug microemulsion using
DLS. Two diffusion models were found in microemulsion. Rapid diffusion model affected the number of droplets, whereas low-diffusion model impacted polydispersity.

\section{Fourier transform infrared spectroscopy (FTIR)}

FTIR is a spectroscopic technology, which uses infrared spectroscopy to identify and analyze molecules. Water in the microemulsion system has three states: combined water, bound water, and free water. Different states of water have different thermodynamic and spectrum properties. Araújo et al. ${ }^{[17]}$ reported the self-assembly of thalidomide microemulsion, with the analysis of thalidomide crystals during crystallization process using FTIR. They identified different crystal forms in this system. Gang Zhou et al. ${ }^{[18]}$ prepared and characterized starch nanoparticles in ionic liquid-in-oil microemulsions system. FTIR results in their study revealed the formation of crosslinking bonds in starch molecules.

\section{E. Magnetic Resonance Imaging (MRI)}

MRI is a powerful and sophisticated analytical tool, which can be used to analyze and quantify the liquid or solid compounds and their structures. MRI can also be used to study the whole complex ${ }^{[19]}$. MRI is able to evaluate the self-diffusion coefficient of each component of microemulsion system. In oil-in-water microemulsion, self-diffusion coefficient of water is smaller than pure water. In water-in-oil microemulsion, oil is dispersed, and self-diffusion coefficient of oil is lower than that of pure oil in the oil phase. Casadei et al. ${ }^{[20]}$ analyzed and identified ibuprofen entrapment state and encapsulation efficiency in solid lipid particles using MRI.

\section{F. Differential scanning calorimetry (DSC)}

DSC is a thermal analysis method. DSC can be used to measure thermal properties and heat changes of different components in the microemulsion ${ }^{[21]}$. DSC can be used to infer the existence state of water in the formation of microemulsions, and to identify the type of microemulsion $^{[22]}$. In order to study microemulsions formation mechanism, Hui Zhang et al. ${ }^{[23]}$ studied U-type antimicrobial microemulsions system using DSC. The DSC results showed that with up to $33 \%$ water, emulsion formed water-in-oil microemulsions. As water content increased, water gained mobility, and transformed into bicontinuous state with $33-39 \%$ water. Finally the microemulsions became oil-in-water with more than $39 \%$ water.

\section{G. Conductivity}

Conductivity is an important attribute of microemulsions. Conductivity reflects the structure and properties of microemulsions ${ }^{[24]}$ Oil-in-water microemulsions have high conductivity, because water is at continuous phase with a high conductivity. Water-in-oil microemulsions have low conductivity because the relatively poor conductivity of the oil. Significant change of conductivity will happen during the transform between oil-in-water and water-in-oil structures. Therefore, plotting conductivity curve is helpful to study the structure of microemulsion. Zhang Hui et al. ${ }^{[23]}$ used DSC and conductivity method to characterize food-grade Umicroemulsion. The results showed that thermal transition coincided with structural transitions by electrical 
conductivity measurements. They found that that structural transition occurred at 35 and 65 mass percentage of water during dilution. Qiong Zhou et al. ${ }^{[25]}$ focused on understanding of the relationship between morphology, crystallinity and conductivity of PANI powder. As water content increased in the microemulsion system, morphology and crystallinity significantly changed.

\section{CONCLUSIONS}

Microemulsions are highly dispersed, stable and easy to prepare. Microemulsions have great applications in food products to carry low soluble and instable hytotherapeutic, nutraceuticals and food additives. In this report, preparation and characterization methods of microemulsions were summarized. It is anticipated that an increasing number of new technologies will be applied to synthesize and investigate food-grade microemulsions.

\section{ACKNOWLEDGMENT}

We gratefully acknowledge the financial support of the Natural Science Foundation, Education Department of Heilongjiang Province, China (Grant C201333), and Qiqihar university graduate innovation research projects (Grant YJSCX2014-014X). The authors would like to thank the Key Laboratory of Agricultural Products Processing of Provincial University, Qiqihar University, for providing laboratory facilities.

\section{REFERENCES}

[1] Badawi A A, Nour S A, Sakran W S, El-Mancy S M S. Preparation and evaluation of microemulsion systems containing salicylic acid[J]. AAPS PharmSciTech. 2009,10(4):1081-1084.

[2] Hoar T, Schulman J. Transparent water-in-oil dispersions: the oleopathic hydro-micelle[J]. Nature. 1943,152:102-103.

[3] Schulman J H, Stoeckenius W, Prince L M. Mechanism of formation and structure of micro emulsions by electron microscopy[J]. The Journal of Physical Chemistry. 1959,63(10):1677-1680.

[4] Salimi A, Zadeh B S M, Moghimipour E. Preparation and Characterization of Cyanocobalamin (Vit B12) Microemulsion Properties and Structure for Topical and Transdermal Application[J]. Iranian journal of basic medical sciences. 2013,16(7):865.

[5] Flanagan J, Singh H. Microemulsions: a potential delivery system for bioactives in food $[\mathrm{J}]$. Critical reviews in food science and nutrition. 2006,46(3):221-237.

[6] Chen L, Tan F, Wang J, Liu F. Assessment of the percutaneous penetration of indomethacin from soybean oil microemulsion: effects of the HLB value of mixed surfactants[J]. Die Pharmazie-An International Journal of Pharmaceutical Sciences. 2012,67(1):31-36.

[7] McIntosh T J, Lin H, Li S, Huang C-h. The effect of ethanol on the phase transition temperature and the phase structure of monounsaturated phosphatidylcholines $[\mathrm{J}]$. Biochimica et Biophysica Acta (BBA)-Biomembranes. 2001,1510(1):219-230.

[8] Groot R D. Mesoscopic simulation of polymer-surfactant aggregation[J]. Langmuir. 2000,16(19):7493-7502.
[9] Rekvig L, Kranenburg M, Hafskjold B, Smit B. Effect of surfactant structure on interfacial properties[J]. EPL (Europhysics Letters). 2003,63(6):902.

[10] TIAN Y, XIE X a, LI Y, LI L, PAN Y, HE K. Mesoscopic simulation of microstructure changes in U-type microemulsion with water phase solubilizing[J]. CIESC Journal. 2012,6:025.

[11] Curry A, Appleton H, Dowsett B. Application of transmission electron microscopy to the clinical study of viral and bacterial infections: present and future[J]. Micron. 2006,37(2):91-106.

[12] Chu B-S, Ichikawa S, Kanafusa S, Nakajima M. Preparation of protein-stabilized $\beta$-carotene nanodispersions by emulsificationevaporation method $[\mathrm{J}]$. Journal of the American Oil Chemists' Society. 2007,84(11):1053-1062.

[13] Yang H, Yang H-m, Kong P, Zhu Y-m, Dai S-g, Zheng G. Concentration measurement of particles by number fluctuation in dynamic light backscattering[J]. Powder Technology. 2013,246:499-503

[14] Salazar-Alvarez G, Björkman E, Lopes C, Eriksson A, Svensson S, Muhammed M. Synthesis and nonlinear light scattering of microemulsions and nanoparticle suspensions $[\mathrm{J}]$. Journal of nanoparticle research. 2007,9(4):647-652.

[15] Zielińska K, Pietkiewicz J, Saczko J, Wilk K A. Microemulsions Stabilized by Gemini, Dicephalic and Single-Head Single-Tail Sugar Surfactants as Biologically Important Systems: Hemolytic Activity and Cytotoxic Studies. Trends in Colloid and Interface Science XXIV: Springer; 2011. p. 193-196.

[16] Shukla A, Neubert R H. Diffusion behavior of pharmaceutical O/W microemulsions studied by dynamic light scattering $[\mathrm{J}]$. Colloid and Polymer Science. 2006,284(5):568-573.

[17] Araújo F, Kelmann R, Araujo B, Finatto R, Teixeira H, Koester L. Development and characterization of parenteral nanoemulsions containing thalidomide[J]. European Journal of Pharmaceutical Sciences. 2011,42(3):238-245.

[18] Zhou G, Luo Z, Fu X. Preparation and characterization of starch nanoparticles in ionic liquid-in-oil microemulsions system[J]. Industrial Crops and Products. 2014,52:105-110.

[19] Rouessac F, Rouessac A. Chemical analysis: modern instrumentation methods and techniques[M]: John Wiley \& Sons; 2013.

[20] Casadei M A, Cerreto F, Cesa S, Giannuzzo M, Feeney M Marianecci C, et al. Solid lipid nanoparticles incorporated in dextran hydrogels: a new drug delivery system for oral formulations $[\mathrm{J}]$. International journal of pharmaceutics. 2006,325(1):140-146.

[21] Venturini C G, Jäger E, Oliveira C P, Bernardi A, Battastini A M, Guterres S S, et al. Formulation of lipid core nanocapsules[J]. Colloids and Surfaces A: Physicochemical and Engineering Aspects. 2011,375(1):200-208.

[22] Sadurní N, Solans C, Azemar N, García-Celma M J. Studies on the formation of $\mathrm{O} / \mathrm{W}$ nano-emulsions, by low-energy emulsification methods, suitable for pharmaceutical applications[J]. European Journal of Pharmaceutical Sciences. 2005,26(5):438-445.

[23] Zhang H, Taxipalati M, Que F, Feng F. Microstructure characterization of a food-grade U-type microemulsion system by differential scanning calorimetry and electrical conductivity techniques[J]. Food chemistry. 2013,141(3):3050-3055.

[24] HUANG M, SUN H, MA W, LIU S, YANG G, HU X. Study on the Four-component Microemulsion System of Cetyltrimethyl Ammonium Bromide/n-Butanol/Cyclohexane/Water by Electroconductivity[J]. Materials Review. 2007:S1.

[25] Zhou Q, Wang J, Ma Y, Cong C, Wang F. The relationship of conductivity to the morphology and crystallinity of polyaniline controlled by water content via reverse microemulsion[J]. Colloid $\begin{array}{lll}\text { and } & \text { Polymer } & \end{array}$ 